\title{
Smart-Clothing Wireless Flex Sensor Belt Network for Foetal Health Monitoring
}

\author{
Luís M. Borges, Norberto Barroca, Fernando J. Velez \\ Instituto de Telecomunicações - DEM, \\ Universidade da Beira Interior, \\ Covilhã, Portugal \\ lborges@1x.it.pt, norberto.barroca@hotmail.com \\ fjv@ubi.pt
}

\begin{abstract}
In the past years low power circuits design and networking techniques not only reduce the total power, requirements for Wireless Sensor Networks (WSNs) but also allow for supporting more complexity. In this paper we present solutions for WSN applications, and design aspects in the context of patient monitoring. The solution presented whose primary function is to collect the vital data remotely from the various sensors in low-rate wireless personal area network (LR-WPAN) is based on the IEEE 802.15.4 standard. It also will includes a WiFi Layer in the context of hybrid networks. The application being dealt here consist of several flex sensors attached to a wearable monitoring belt, and allows for monitoring the foetal movements for a pregnant woman.
\end{abstract}

Keywords- WSNs; Foetal health; Smart clothing; patient monitoring

\section{INTRODUCTION}

WSNs have open a new world of opportunities in the context of healthcare monitoring, and the recent developments in microelectronics have made the creation of small wireless nodes that operate together creating a well defined multi-hop network possible. In near future, new systems capable to be integrated into specialized medical wearable technology will be made available through pervasive wireless sensor networks. Research efforts are therefore needed on the integration of functional textile materials and wireless communication networks in the context of human body while statistical methods for the data analysis and treatment need to be developed, as described in [1].

One of the primary objectives of the Smart Clothing for Health Monitoring and Sport Applications (Smart-Clothing) project [2] is to monitoring the foetal movement in the last weeks of pregnancy through wearable solutions where the sensors are integrated. Wireless solutions have been explored whose purpose is to allow for freedom of movements at home or in the Hospital.

The basic ideas is to expand the pregnant woman movements beyond a single location, and produce a prototype for a easy to wear gear so with this paper we will purpose some of the options available for these interconnections, and the terminology used to discuss them. Hybrid communications can be a solution to obtain a network of networks, e.g., by using Internet Protocol IP (Internet Protocol) it upper layer. A bottom-up architecture formed by i) WSNs low-rate wireless personal area network (LR-WPAN) and ii) Wi-Fi, paving the

\author{
António S. Lebres \\ Unidade de Detecção Remota - Departamento de Física \\ Universidade da Beira Interior \\ Covilhã, Portugal \\ lebres@ubi.pt
}

way for future healthcare monitoring anyway, anywhere and anytime.

The monitoring of the foetal health in the periods between the medical sessions during the last weeks of low risk pregnancies is based in traditional protocols for counting the foetal movements felt by the mother [3]. One example of how this theme of pregnancy monitoring is gaining importance in the research community is mentioned in [4], which describes an innovative remote monitoring decision support system, used in the early diagnosis of pregnancy complications, through the effective and non-invasive monitoring of maternal and foetal electrocardiograms. Besides, some patents on foetal monitoring are presented in [5], [6], [7].

In the context of WSN used in medical applications is important not only to create a device platform that should accommodate a wide range of sensors, depending on needs, expendability and cost, but we need to create a reliable mean to share the information across the network so the remote agents can be either collectors of information (by storing the data in a database that could be accesses later on) or intervenient in a real time monitoring process such for example a nurse or a doctor that is monitoring the foetus in the pregnant women, all this information is available by the use of a Mote Interface Board (Gateway) that is attached to a PC.

The structure of this paper is as follows. Section II describes the solution proposal for remote monitoring. It includes the presentation of the architecture and a possible scenario. Section III analyses the standalone solution of the flex sensor belt. Section IV presents the results of our wireless flex sensor belt sensor network. Finally, conclusions and suggestion for future work are presented in Section V.

\section{REMOTE MONITORNG}

\section{A. Concept}

In order to perform remote monitoring some application scenarios where defined in [1]. Here, the proposed prototype involves a Flex sensor, which has a variable printed resistor Fig. 1.

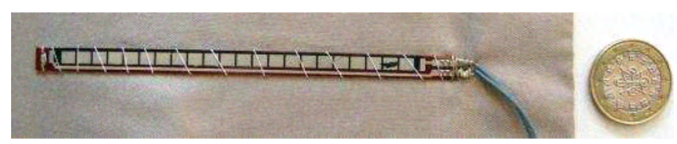

Fig. 1. Flex sensor incorporated in the Smart-Clothing belt. 
The bent of the substrate of this type of sensor produces a resistance output correlated to the bend radius. A belt was produced that accommodates five Flex sensors. With this belt, it is possible to find the deformation angles caused by the foetus movements. Figure 2 represents a simplified block diagram of the system. The deformation angles are collected and data is transmitted across the IEEE 802.15.4 network.

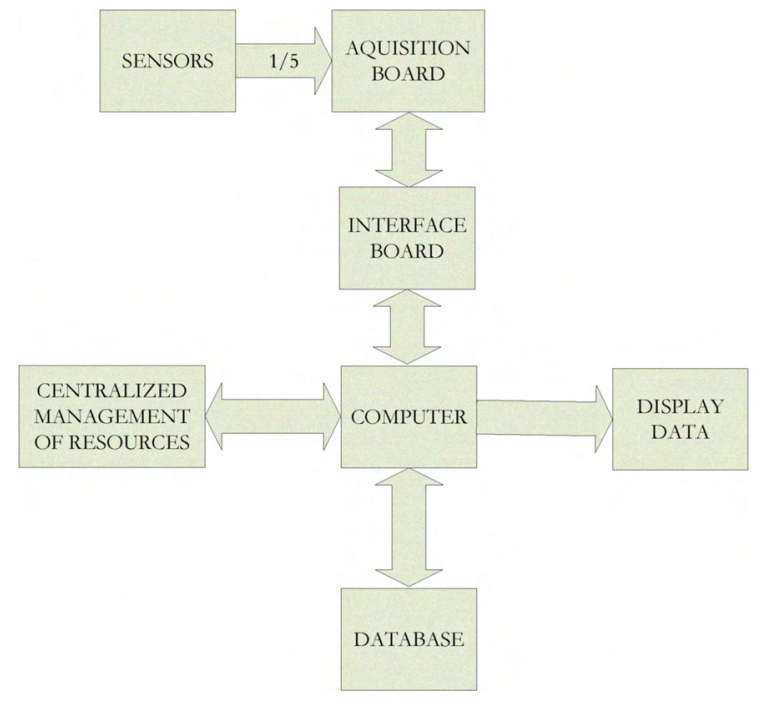

Fig. 2. Block Diagram for the acquisition system.

The IEEE 802.15.4 sensor network, whose primary function is to collect the vital data remotely, from the various sensors that are applied onto the patient body, allows for monitoring the foetal movements of a pregnant woman while transmitting the data to a Mote Interface Board (Gateway) that is directly connected to our Centralized Management of Resources (CMR) entity.

A previous version of the belt incorporated eight Flex sensors but did not have wireless communication capabilities. As three ADC channels are already connected to inbuilt sensors from the mote board only five channels remain available.

\section{B. Wireless networking architecture}

The Centralized Management of Resources is formed by a base station, a personal computer, an application that is responsible to present the data (and save all the records in a database), and a Wi-Fi module, to transmit data across a WLAN, as shown in Fig. 3.

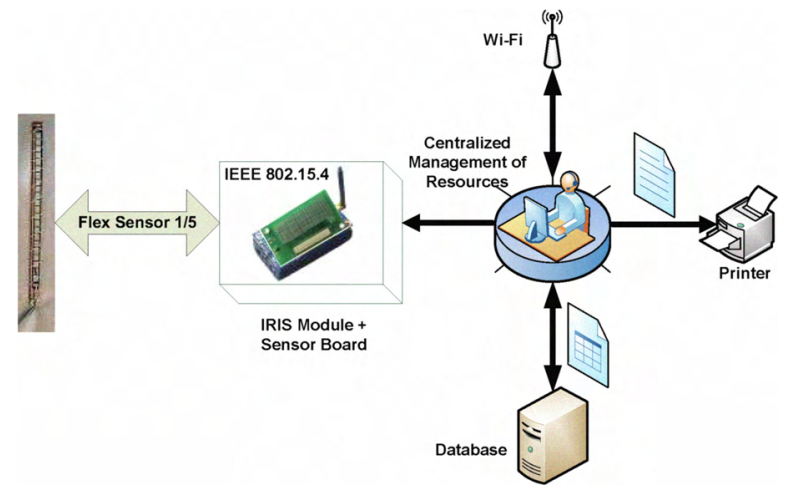

Fig. 3. Patient Monitoring using IEEE 802.15.4 and a Wi-Fi Wireless networking architecture.
In our prototypes, IRIS Motes are used, forming a $2.4 \mathrm{GHz}$ IEEE 802.15.4 tiny wireless measurement system, designed specifically for embedded sensor networks [8].

The IRIS Mote is one of the components of the MICA family (model XM2110CA). Its primary characteristics are very similar to the MICAZ, with the same data transmission rate, and radio frequency band, as the previous model. The system supports TinyOS and is fully compatible with accessories for the MICA family. It is suitable for applications that require high speed in data transmissions as, for example, real time reading from a sensor in Indoor Building Monitoring. The main advantages are the following: range up to 500 meter (between nodes) without the need to amplify the signal, and $8 \mathrm{kB}$ of RAM, almost the double than the existing modules in the past.

Figure 4 presents a possible scenario on a Hospital floor. The Centralized Management of Resources is located in a convenient location, and the red spots represent the pregnant women with the belt. This Figure represents the communication system employed to deliver the data from the Wireless Body Area Network (WBAN) that is installed in the pregnant woman.

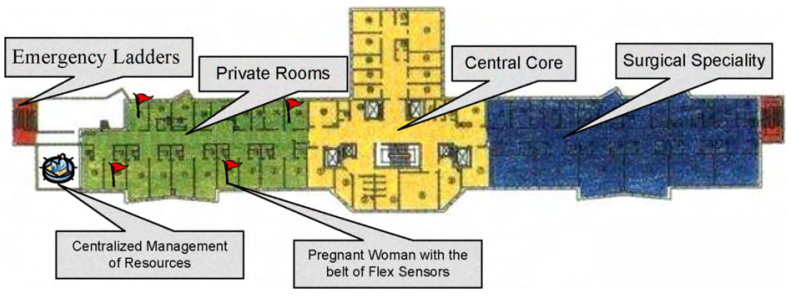

Fig. 4. Possible Monitoring using IEEE 802.15.4 in a Hospital.

Note that we can easily move our Centralized Management of Resources to another part or the Hospital; hence we are not limited by the geographical position of any of the components of the system.

Since the system is still in the prototype phase, interference robustness was not yet taken into account. Tests were performed in laboratorial environment where several wireless networks coexist, and no problems were detected so far.

\section{STANDALONE FLEX SENSOR BELT}

To operate with the Flex sensor a simple voltage divider was enough. A resistance value of $10 \mathrm{k} \Omega$ matches an angle of $0^{\circ}$ while a value of $14 \mathrm{k} \Omega$ matches an angle of $90^{\circ}$ and a value of $22 \mathrm{k} \Omega$ matches an angle of $180^{\circ}$. Data on the mapping between the resistance and angles is supplied by the sensor manufacturer. For the resistance value obtained from the flex sensor, by using interpolation equations, it is possible to calculate the corresponding bent angle.

The associated system diagram is presented in Fig. 5, where just one Flex sensor is presented. However, all eight sensors were connected to a single voltage divider. Besides the Flex sensor, a button was incorporated to be pressed by the pregnant woman when she feels or detects the foetus moving. These events are very useful for comparison purposes, as they enable a comparison with the movements detected automatically by the belt. An initial drawing of this system had the power supply connected to each voltage divider, but 
when the system was turned on the system failed because of not supporting enough current to hold on all the voltage dividers as well as the microcontroller.

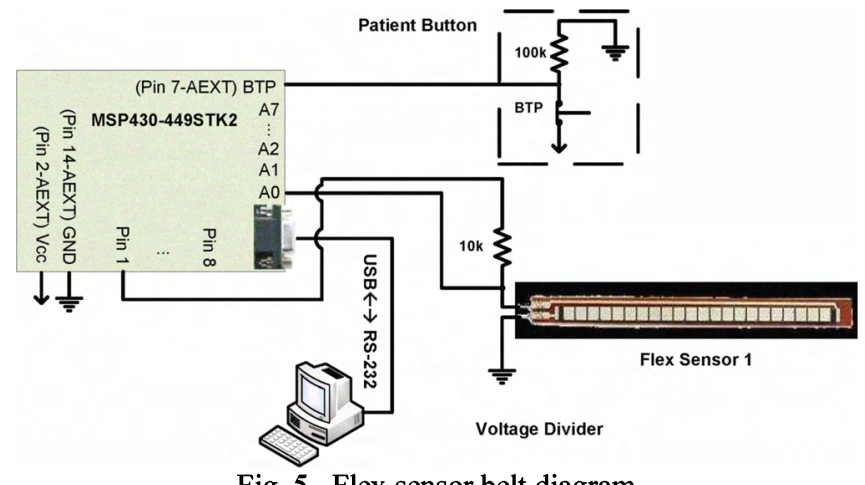

Fig. 5. Flex sensor belt diagram.

To overcome this limitation, the solution was to supply the $V_{c c}$ voltage to the voltage divider one each time in a sequence that takes only $100 \mathrm{~ms}$, by using external pin from the microcontroller and read the voltage from the voltage divider using the Analog-to-Digital Converter (ADC) from the microcontroller. To compute the value of the resistance from the Flex sensor one uses the voltage divider formula, as follows

$$
R_{\text {Flex } 1}=\frac{R_{1}\left(V_{\text {in }}-V_{\text {out }}\right)}{V_{\text {out }}},
$$

where $R_{\text {Flex } 1}$ is the resistance value, $R_{1}$ is equal to $10 \mathrm{k} \Omega, V_{\text {in }}$ is the $V_{c c}$ value supplied from the Pin 1 of the microcontroller to the voltage divider, and $V_{\text {out }}$ is the voltage value from the voltage divider which is the connected to the channel A0 of the ADC. The $V_{c c}$ voltage supplied to the voltage divider is measured every 100 measurements by a routine that is located into the microcontroller, in order to compensate the battery losses during the system operation and to achieve a better accuracy of the values from the Flex sensors.

For the conversion of the resistance value to the angle value two formulas were used

$$
\begin{gathered}
\theta_{1}\left[^{\circ}\right]=\frac{R_{\text {Flex } 1}-10^{*} 10^{3}}{44,44}, \\
\theta_{2}\left[^{\circ}\right]=\frac{R_{\text {Flex } 1}-6001}{88,88},
\end{gathered}
$$

where $\theta_{1}$ and $\theta_{2}$ are the angles for the corresponding Flex sensor resistance value.

Equation (2) is used when the resistance value is between $10 \mathrm{k} \Omega$ and $14 \mathrm{k} \Omega$ while equation (3) is used when the resistance value is between $14 \mathrm{k} \Omega$ and $22 \mathrm{k} \Omega$.

\section{RESULTS}

The application presented in Fig. 6 is responsible for making all data collected by the sensors available to the nurse/doctor, in real time. So, this will be one additional tool available for diagnostic.

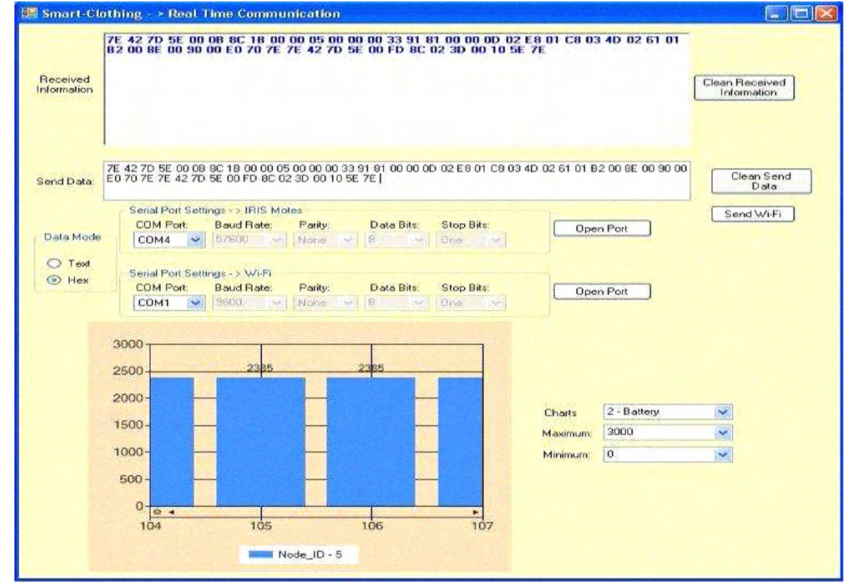

Fig. 6. Smart Application Real Time Communication.

In Fig. 6, by using our application alone when a reading from the Flex Sensor 1 is performed the data is shown in real time. Besides, reading the data directly from the "emulated" serial port, the application presents it to the user after some processing (that distinguishes not only the size of received frames but also the sequence for data reading because the data is scrambled).

Besides reading the data from the five Flex Sensors there is other set of parameters that can be monitorized. Examples are battery voltage, ambient temperature, and the light of the room. The first parameter is very important because we need to know the state of the batteries and change them if it is needed. Besides, we may monitor the energy consumption, and propose new algorithms based on energy consumption reduction. The purpose is to try and optimize the trade-off between energy consumption/processing and communication capabilities. The sampling frequency is 1 sample/s.

For the standalone flex sensor belt, tests were performed with a patient that was not a pregnant woman. The objective was to verify if the respiration movements or other type of motion artifact, influence the angles of each Flex sensor in the belt. It was verified that the respiration movements were slightly felt. However, if the patient moves quickly then the sensors could detect the deformation from the belt. After these preliminary tests, the belts need to be tested in a pregnant woman, in order to detect the foetus movements. These occurrences will be compared with the one when the pregnant woman presses the button (but the system does not detect the movement).

By considering these initial tests, a suggestion is to implement a routine which automatically defines the value for a detection threshold-trigger whose values will be tuned. Figure 7 presents the application to display the sensors deformation angles. For each sensor, an independent threshold trigger can be defined individually, or a unique threshold value can be defined as a whole for all the sensors. With this thresholdtrigger even if the sensor detects some motion artifact a boundary can be established in order to tune when it should count the deformation angle as a foetal movement. In Fig. 8 the value used for the global threshold is equal to 15 degree for angle deformation. 


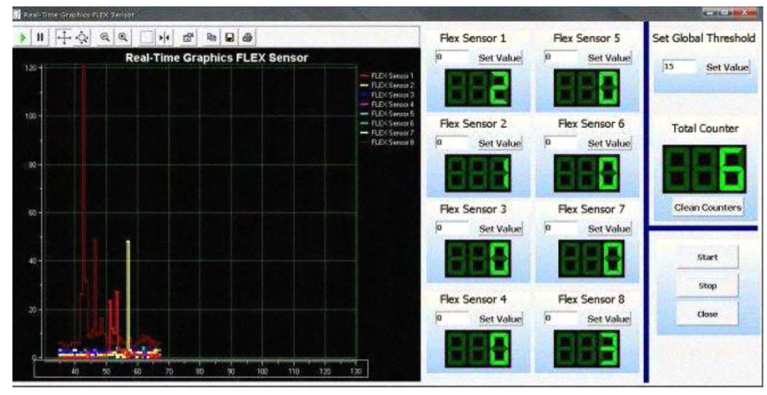

Fig. 7. Graphical application to display the deformation angles.

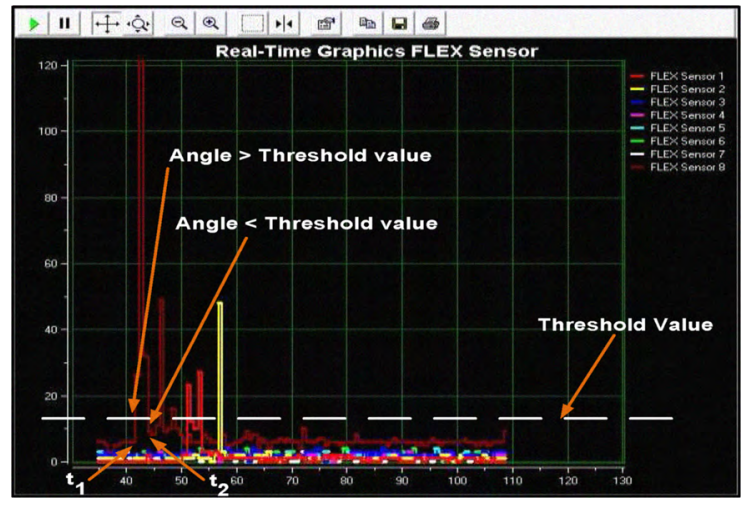

Fig. 8. Real-time graphics Flex Sensor plot.

This means that the automatic counter from each Flex sensor counts a movement when the instant value of the Flex sensor angle is larger than the threshold value in one time instant and smaller in the next time instant. As an example of how the automatic counter works is presented. Considering the Flex sensor 8 in the graphic in time instant $t_{1}$ the angle value is equal to 28 degrees so the counter will count if this value decreases in the next time instant. In time instant $t_{2}$ the angle value is equal $\mathbf{8}$ degrees so the automatic counter will add one unit to the counter of the Flex sensor 8 .

Theoretically, the system performance will be affected if the number of used sensors increases in a way that the system has to spend more time to read from all the sensors (while maintaining the timer value to sweep the voltage output from the sensors). If the timer value for sweeping the flex sensors voltage output is decreased the performance may be worst because the ADC will not have sufficient time to do the conversion, leading to errors.

\section{CONCLUSIONS}

In this work a prototype that allows the continuous monitoring of the foetal health was developed. This was accomplished by using an easy to wear belt and a telemedicine system that will allow remotely monitoring in low risk pregnancies.
We present two versions of Flex Sensor Belts produced within the Smart-Clothing project, where the main objective is counting the movements of the foetus from a pregnant woman.

To accomplish the remote monitoring, a system was created by using a belt with several flex sensors connected to the pregnant woman. It detects the foetal movement based on the bending of the sensors, and an IEEE 802.15.4 network was created so we can deliver all the data collected by the motes to our Centralized Management of Resources. An application is responsible to show the data to the user (nurse/doctor) and save all the records in a database. If there is a need, it is also possible to transmit the data via $\mathrm{Wi}-\mathrm{Fi}$, so the information can be shared or accessed by other people.

In the future, besides real-time data, the CMR will be able to provide long-term statistical information. This solution will answer to the needs of the market, especially in medical applications. Nowadays there are increasing demands on remote monitoring, and on site monitoring is becoming more and more important. It allows for easy access to instantaneous information; wireless devices can therefore help to increase overall efficiency, and to enable better remote patient care.

\section{ACKNOWLEDGMENT}

This work was supported by UDR (Unidade de Detecção Remota), Department of Physics from University of Beira Interior, by Instituto de Telecomunicações, by IST-UNITE, by the PhD FCT (Fundação para a Ciência e Tecnologia) grant SFRH / BD / 38356 / 2007, by Fundação Calouste Gulbenkian, by "Projecto de Re-equipamento Científico" REEQ/1201/EEI/ 2005 (a Portuguese Foundation for Science and Technology project), by the Smart Clothing project. Special thanks go to Ms. Andreia Rente and to Prof. Luisa Rita Salvado who helped in the belt production.

\section{REFERENCES}

[1] Luís M. Borges, Andreia Rente, Fernando J. Velez, Luisa R. Salvado, António S. Lebres, J. Martinez Oliveira, Pedro Araújo, João Ferro, "Overview of Progress in Smart-Clothing Project for Health Monitoring and Sport Applications," in Proc. of ISABEL 2008 - First International Symposium on applied Sciences in Biomedical and Communication Technologies, Aalborg, Denmark, Oct. 2008.

[2] http://www.e-projects.ubi.pt/smart-clothing/

[3] C. Gribbin, D. James, "Assessing fetal health", Current Obstetrics \& Gynaecology, Elsevier, 15, 221-227, 2005.

[4] Evaggelos Cz. Karvounis E., Papaloukas C., Tsipouras M., Bougia P., Dimitrios I., Naka K., "Remote maternal and fetal health monitoring during pregnancy", ITAB2006(IEEE), Ioannina, Greece, 2006.

[5] I Maternal Fetal Monitoring System, US 7,333,850 B2, Feb.19, 2008.

[6] Fetal Wellbeing Monitoring Apparatus and Pad Therefor, WO 2008/010215 A2.R. Nicole, "Title of paper with only first word capitalized," J. Name Stand. Abbrev., in press.

[7] Fetal Wellbeing Monitoring Apparatus and Pad Therefor, WO 2008/010215 A2.

[8] http://www.xbow.com/Products/Product_pdf_files/Wireless_pdf/IRIS_D atasheet.pdf. 